\title{
La maladie de Lyme : Diagnostic clinique et traitement
}

\author{
Hatchette $\mathrm{TF}^{1,2,3^{\star}}$, Davis $\mathrm{I}^{1,2}$ et Johnston $\mathrm{BL}^{2}$ \\ 1 Département de pathologie et de médecine de laboratoire, Régie régionale de la santé Capital (CDHA), Halifax (Nouvelle- \\ Écosse) \\ 2 Département de médecine CDHA et Université Dalhousie, Halifax (Nouvelle-Écosse) \\ 3 Département de pathologie, Université Dalhousie, Halifax (Nouvelle-Écosse) \\ * Auteur-ressource : Todd. hatchette@cdha. nshealth.ca
}

\section{Résumé}

Contexte : La maladie de Lyme est une infection zoonotique émergente au Canada. À mesure que la tique Ixodes agrandit son territoire, de plus en plus de Canadiens seront exposés à Borrelia burgdorferi, la bactérie responsable de la maladie de Lyme.

Objectif : Tour d'horizon du diagnostic clinique et du traitement de la maladie de Lyme à l'intention des cliniciens de première ligne.

Méthodes : Recherche documentaire, limitée aux articles publiés en anglais entre 1977 et 2014, effectuée au moyen de PubMed.

Résultats : Le risque de contracter la maladie de Lyme est plus élevé dans les régions d'endémie, mais toutes les morsures de tiques ne transmettent pas la maladie. Le diagnostic est essentiellement clinique. Les patients peuvent être atteints d'une maladie au stade précoce caractérisée par une éruption cutanée concentrique, une fièvre et des douleurs musculaires ou d'une maladie au stade disséminé précoce qui peut se traduire par des douleurs articulaires, des troubles de la conduction cardiaque ou des symptômes neurologiques. En Amérique du Nord, la maladie de Lyme au stade tardif se manifeste généralement par une oligo-arthrite, mais la présence d'une encéphalopathie subaiguë est également possible. L'antibiothérapie est efficace contre la maladie de Lyme, surtout lorsqu'elle est instaurée au début de l'infection. La doxycycline peut être indiquée en prophylaxie dans certains cas. Bien que des symptômes persistants puissent être observés chez une minorité de patients, rien ne prouve que l'administration de cures prolongées d'antibiotiques améliore le pronostic de la maladie.

Conclusion : Il est impératif que les cliniciens sachent reconnaître les signes et les symptômes de la maladie de Lyme. Il est important de connaître les régions où l'infection à Borrelia est endémique en Amérique du Nord pour déterminer quels sont les patients à risque et les besoins de traitement.

\section{Introduction}

La maladie de Lyme est la zoonose à transmission vectorielle la plus répandue en Amérique du Nord. Aux ÉtatsUnis, il est estimé que plus de 300000 personnes sont infectées chaque année par Borrelia burgdorferi, la bactérie responsable de la maladie (1). Peu de données sont disponibles en ce qui concerne le Canada où la maladie de Lyme n'est une maladie à déclaration obligatoire à l'échelle nationale que depuis 2009. En 2012, 315 cas d'infections ont été signalés au pays, un chiffre qui représente probablement une sous-estimation et comprend les cas provenant d'infections contractées lors de voyages dans d'autres régions d'endémie, comme en Europe (2-4).

Les tiques Ixodes, vecteurs de Borrelia, ont considérablement étendu leur territoire ces vingt dernières années, faisant de la maladie de Lyme une infection émergente au Canada. Des tiques Ixodes ont été identifiées en Colombie-Britannique, dans le sud-est du Manitoba, dans le sud et l'est de l'Ontario, et dans certaines régions du Nouveau-Brunswick, de la Nouvelle-Écosse et du Québec $(4,5)$. À mesure que les tiques Ixodes élargissent leur territoire, de plus en plus de Canadiens seront exposés à $B$. burgdorferi et les cliniciens devront posséder 
les compétences requises pour prendre en charge les morsures de tiques ainsi que diagnostiquer et traiter la maladie de Lyme.

La prise de conscience de la fréquence de la maladie de Lyme à l'échelle locale, la détection et le traitement précoces de la maladie sont des aspects importants de la prise en charge de cette infection émergente. L'objectif du présent article est de faire un tour d'horizon du diagnostic clinique et du traitement de l'infection à $B$. burgdorferi à l'intention des cliniciens de première ligne.

\section{Méthodes}

Une recherche documentaire, limitée aux articles publiés en anglais entre 1977 et 2014, a été effectuée au moyen de PubMed. Les termes de recherche suivants ont été utilisés : Lyme disease and treatment (maladie de Lyme et traitement), Lyme disease and arthritis (maladie de Lyme et arthrite), Lyme disease and clinical presentation (maladie de Lyme et tableau clinique), Lyme disease ticks and risks (maladie de Lyme, tiques et risques), Lyme disease and pregnancy (maladie de Lyme et grossesse), neuroborreliosis (neuroborréliose), chronic Lyme OR post-Lyme syndrome (syndrome de maladie de Lyme chronique OU syndrome post-maladie de Lyme) ET treatment (traitement) (essais cliniques). Des résumés ont été examinés pour y recenser des articles pertinents et les articles mis en évidence par la recherche initiale ont permis d'obtenir d'autres références. Bien qu'il existe, en Amérique du Nord et en Europe, différentes souches de Borrelia associées à des tableaux cliniques qui sont distincts, mais se recoupent, le présent exposé met l'accent sur les manifestations cliniques observées au Canada. Son but n'est pas de décrire les divers aspects des tests diagnostiques, qui font l'objet d'un article complémentaire (6).

\section{Observations}

\section{Qui est à risque de contracter la maladie de Lyme et pourquoi?}

Pour contracter l'infection, il faut avoir subi la morsure d'une tique porteuse de $B$. burgdorferi. Bien que des tiques infectées puissent être transportées de façon intermittente dans différentes régions du Canada par des oiseaux migrateurs, le risque de contracter la maladie de Lyme est le plus élevé dans les régions où les tiques sont présentes de façon persistante ou endémique et dépend de la proportion de tiques infectées. Même dans les régions où la présence des tiques est devenue endémique, la proportion de tiques infectées par le spirochète en cause varie et évolue. Il est conseillé aux cliniciens de se renseigner auprès de leur service local de santé publique sur l'endémicité de la maladie dans la région où ils exercent. Étant donné que la maladie de Lyme est endémique dans d'autres régions de l'Amérique du Nord et en Europe et qu'une partie des cas documentés au Canada sont attribuables à des infections contractées en voyage, il est important d'obtenir un énoncé détaillé des antécédents de voyage des patients. Il est en outre important de transmettre ces antécédents de voyage ou tout soupçon d'infection par une souche européenne de Borrelia au laboratoire de diagnostic, car les épreuves de confirmation ne sont pas les mêmes pour les souches d'Europe et celles d'Amérique du Nord.

\section{Quel est le risque de contracter la maladie de Lyme après une morsure de tique?}

Malgré le nombre et l'envergure limités des études réalisées, les données provenant des États-Unis et d'Europe donnent à penser que le risque de contracter la maladie de Lyme, même après la morsure d'une tique infectée, est faible, de l'ordre de < $1 \%$ à $6 \%$ (7). Dans des études menées pour évaluer l'efficacité de la prophylaxie antibiotique chez des personnes ayant subi une morsure de tique dans des régions où la maladie de Lyme est endémique (prévalence de tiques porteuses de B. burgdorferi située entre 15 et $50 \%$ ), le taux d'infection parmi les personnes ayant reçu le placebo se situait entre 1,1 et 3,4\% (8-11). Ces taux sont conformes à ceux relevés dans le cadre d'une étude prospective menée auprès de patients ayant subi une morsure de tique dans une région de l'État de New York où la maladie de Lyme est endémique, selon laquelle l'infection s'était déclarée chez $3,7 \%$ des personnes mordues (12).

Le risque d'infection consécutif à la morsure d'une tique est lié à la durée pendant laquelle la tique reste accrochée et s'explique par le cycle de vie de Borrelia. Après la morsure d'une tique, le spirochète doit tout d'abord migrer de l'intestin de la tique vers ses glandes salivaires avant de pouvoir être injecté dans l'organisme 
de la personne mordue. Pour ce faire, la structure antigénique de la membrane externe de la bactérie doit subir un changement favorable à l'infection de l'humain (13-16). Ce processus prend environ 36 heures (14). Si la tique est retirée pendant cette période, l'infection est presque toujours évitée. Dans une étude évaluant une prophylaxie antibiotique menée aux États-Unis, aucune infection n'a été observée chez les participants des groupes ayant reçu le placebo ou la prophylaxie lorsque la tique s'était nourrie pendant moins de 72 heures (11). Ces constatations sont étayées par les données d'une autre étude prospective menée dans une région d'endémie, selon lesquelles le risque d'infection était plus élevé lorsque la tique était restée attachée plus de 72 heures (12).

\section{Dans quel cas l'administration d'une prophylaxie est-elle indiquée après une morsure de tique?}

La décision de proposer ou non une prophylaxie après une morsure de tique doit reposer sur la prise en compte d'un certain nombre de facteurs (tableau 1). Tous les essais visant à évaluer la prophylaxie ont été menés dans des régions où la maladie de Lyme serait considérée comme hyperendémique, où le fardeau de l'infection par les tiques est supérieur à $20 \%$ et où les antibiotiques étaient administrés dans les 72 heures du retrait de la tique (8-11). Selon les données regroupées d'une méta-analyse préliminaire sur l'administration prophylactique d'antibiotiques en prévention de la maladie de Lyme, le taux d'infection était de 1,4\% chez les patients ayant reçu le placebo et de $0 \%$ chez ceux ayant reçu un antibiotique (17). En raison de leur petite échelle, les essais inclus dans la méta-analyse n'ont pas permis de démontrer un quelconque effet significatif de la prophylaxie, vu la faible incidence de l'infection parmi les sujets ayant reçu le placebo (8-10). Un essai ultérieur, à répartition aléatoire et contrôlé par placebo, a démontré qu'une seule dose de doxycycline pouvait réduire le taux de maladie de Lyme (11).

Tableau 1. Critères de décision et prophylaxie recommandée après une morsure de tique (19)

\section{Critères d'administration d'une prophylaxie*}

1. La tique accrochée peut être identifiée de façon fiable comme étant une tique à pattes noires (tique à chevreuil/Ixodes scapularis) accrochée depuis une durée estimée à plus de 36 heures d'après son degré d'engorgement ou d'après le moment de la morsure s'il est connu.

2. La prophylaxie peut être instaurée dans les 72 heures du retrait de la tique.

3. Le taux d'infection des tiques par B. burgdorferi dans la région est supérieur à $20 \%$ (se renseigner auprès des autorités locales de santé publique).

4. La doxycycline n'est pas contre-indiquée.

Adultes et enfants $\quad$ Une seule dose de $200 \mathrm{mg}$ de doxycycline administrée par voie
de plus de 8 ans $\quad$ orale $(4,4 \mathrm{mg} / \mathrm{kg}$ pour les patients pesant moins de $45 \mathrm{~kg})$

Enfants de moins $\quad$ Non recommandée de 8 ans et grossesse

* Envisager une prophylaxie antibiotique après la morsure d'une tique lorsque la personne concernée répond à tous les critères.

Selon une nouvelle revue systématique et une méta-analyse, il faudrait administrer une prophylaxie à 49 cas pour prévenir un cas de maladie de Lyme (18). Les patients ayant contracté l'infection ont été traités avec succès et n'ont connu aucune séquelle à long terme. Étant donné que le nombre d'infections observées dans chaque étude était relativement faible et que tous les cas ont été traités avec succès, on peut conclure que la décision de recourir à une prophylaxie antimicrobienne doit tenir compte des effets secondaires potentiels, effets signalés, quoique sans gravité, dans ces études. 


\section{Comment se manifeste la maladie de Lyme chez les patients et comment est-elle} traitée?

Bien que les patients atteints d'une infection à $B$. burgdorferi puissent être asymptomatiques, la plupart des cas de maladie de Lyme se manifestent en l'un de trois stades, les stades pouvant se succéder en l'absence de prise en charge du stade précédent : un stade localisé précoce (en général, moins de 30 jours après l'exposition à la bactérie), un stade disséminé précoce (moins de trois mois après l'exposition) et un stade disséminé tardif (plus de trois mois après l'exposition) (tableau 2).

Tableau 2. Manifestations cliniques de la maladie de Lyme $(19,24,39)$

\begin{tabular}{|c|c|c|}
\hline Stade & Système & Manifestation \\
\hline \multirow[t]{4}{*}{$\begin{array}{l}\text { Stade localisé précoce de la maladie } \\
\text { (<30 jours) }\end{array}$} & Peau & $\begin{array}{l}\text { Érythème migrant (erythema migrans) (remarque : } \\
\text { doit mesurer }>5 \mathrm{~cm} \text { de diamètre, être indolore et se } \\
\text { propager lentement) }\end{array}$ \\
\hline & Systémique & Fièvre \\
\hline & & Douleurs articulaires \\
\hline & & Céphalée \\
\hline \multirow{13}{*}{$\begin{array}{l}\text { Stade disséminé précoce de la maladie } \\
(<3 \text { mois })^{\star}\end{array}$} & Peau & Plusieurs érythèmes migrants \\
\hline & Systémique & Fièvre \\
\hline & & Douleurs articulaires \\
\hline & & Céphalée \\
\hline & & Adénopathie \\
\hline & Cœur & Bloc atrio-ventriculaire \\
\hline & & Tachyarythmies \\
\hline & & Myopéricardite \\
\hline & & Dysfonction myocardique \\
\hline & SNC & Méningite à liquide clair \\
\hline & & $\begin{array}{l}\text { Neuropathie crânienne (en particulier paralysie du } \\
\text { nerf facial) }\end{array}$ \\
\hline & & Radiculopathie, atteinte motrice ou sensorielle \\
\hline & Yeux & Conjonctivite (rare) \\
\hline \multirow{5}{*}{$\begin{array}{l}\text { Stade disséminé tardif de la maladie } \\
(>3 \text { mois })^{\star}\end{array}$} & Musculosquelettique & Oligo-arthrite \\
\hline & SNC & Encéphalopathie \\
\hline & & Polyradiculoneuropathie axonale \\
\hline & & Encéphalomyélite chronique \\
\hline & Yeux & Rétinite (rare) \\
\hline
\end{tabular}

* Remarque : Bien qu'il puisse y avoir des exceptions, ces délais donnent aux cliniciens une idée générale du délai d'apparition des différentes manifestations de la maladie.

$\mathrm{SNC}=$ Système nerveux central.

Le traitement de la maladie de Lyme dépend du stade d'évolution de l'infection et du système d'organes concerné; il est résumé dans des lignes directrices de pratique clinique mises au point par l'IDSA (Infectious Diseases Society of America) (tableau 3) (19). Le tableau clinique de la maladie et certaines des publications à l'appui de ces recommandations de traitement sont résumés dans les sections qui suivent.

Tableau 3. Lignes directrices de pratique clinique de la Infectious Disease Society of America sur le traitement de la maladie de Lyme (19)* 
Traitement des adultes et des enfants de plus de 8 ans atteints de maladie de Lyme
Traitement des enfants de 8 ans ou moins atteints de maladie de Lyme
Érythème migrant ou stade disséminé précoce de la maladie, notamment paralysie de Bell, mais sans autres atteintes du SNC

Stade précoce avec atteintes du SNC

Stade précoce, avec cardite

Stade précoce, avec cardite
.

Stade tardif sans atteintes
du SNC** Stad

Stade tardif avec atteintes du SNC (neuroborréliose tardive)

Stade localisé précoce de la maladie
- Doxycycline, 100 mg, p.o., 2 f.p.j. x 14 à 21 jours (contre indiquée en cas de grossesse)

- Amoxicilline, 500 mg, p.o.,3 f.p.j. x 14 à 21 jours

- Céfuroxime, 500 mg, p.o., 2 f.p.j. $x$ de 14 à 21 jours

- Ceftriaxone, 2 g, i.v. 1 f.p.j. x de 14 à 28 jours

- Pénicilline G $4 \times 10^{6}$ unités i.v. q 4 h x 14 à 28 jours

- Doxycycline, 100 à 200 mg, p.o., 2 f.p.j. x 28 jours (option possible si les autres options ne sont pas envisageables)

- Même traitement que pour le stade précoce avec atteintes du SNC, mais administration initiale par voie intraveineuse en présence d'un blocage cardiaque de grade élevé ou si l'hospitalisation est nécessaire.

- Doxycycline, 100 mg, p.o., 2 f.p.j. x 28 jours

- Amoxicilline, 500 mg, p.o., 3 f.p.j. x 28 jours

- Céfuroxime, 500 mg, p.o., 2 f.p.j. x 28 jours

- Ceftriaxone, 2 g, i.v. 1 f.p.j. x de 14 à 28 jours

- Pénicilline G $4 \times 10^{6}$ unités i.v. q 4 h x 14 à 28 jours

- Amoxicilline $30 \mathrm{mg} / \mathrm{kg}$ par jour, par voie orale, divisée en trois doses (max. de 1,5 g/jour) pendant 14 à 21 jours

- Pour les enfants allergiques à la pénicilline, céfuroxime $30 \mathrm{mg} / \mathrm{kg}$ par jour, par voie orale, en deux doses fractionnées (maximum de $1 \mathrm{~g} / \mathrm{jour}$ ) pendant 14 à 21 jours

- Traitement par voie orale comme le traitement par voie orale ci-dessus pendant 21 jours

stade tardif

plusieurs érythèmes

migrants

Stade disséminé précoce et stade tardif :

paralysie faciale isolée et premiers épisodes d'arthrite

Stade disséminé précoce et stade tardif :

arthrite

persistante/récurrente,

cardite et

méningite/encéphalite

\footnotetext{
Ces recommandations correspondent aux meilleures pratiques fondées sur des données probantes et non pas à des ** indications approuvées.

** CEdème articulaire récurrent ou persistant : répéter la cure d'antibiotiques oraux de 4 semaines selon les indications cidessus. Réserver l'administration i.v. de ceftriaxone au traitement des œdèmes articulaires récurrents ou persistants réfractaires au traitement par voie orale.

SNC $=$ Système nerveux central.
}

\section{Infection asymptomatique}

L'infection peut être asymptomatique chez environ 1,6 à $7 \%$ des personnes infectées $(7,20)$. Le pronostic pour ces patients est généralement favorable. Dans une vaste étude sur la vaccination menée dans dix États des États-Unis où la maladie de Lyme est endémique, des infections asymptomatiques ont été constatées chez $7 \%$ 
(15/269) des participants. Bien que la majorité des participants aient reçu un traitement après constatation de la séroconversion, seule 1 des 8 personnes qui n'avaient pas reçu de traitement a présenté des signes d'arthrite dans les 12 mois suivant la séroconversion (20). Malgré une période de suivi relativement courte, ces données appuient la notion que l'infection asymptomatique ne nécessite pas de traitement et que la maladie finit par se déclarer d'elle-même si l'infection persiste.

\section{Stade localisé précoce de la maladie (< 30 jours)}

Le stade précoce se manifeste généralement par une maladie aiguë caractérisée par une fièvre, une céphalée et une myalgie et la présence d'une lésion cutanée unique localisée appelée erythema migrans (EM, érythème migrant). Cette éruption cutanée caractéristique est présente chez environ $80 \%$ des patients au stade précoce de la maladie et disparaît sans antibiothérapie dans un délai médian de 28 jours $(21,22)$. Bien que chez la plupart des patients l'érythème migrant fasse son apparition dans les sept jours suivant la morsure de tique initiale, la période d'incubation peut varier de 3 à 30 jours (23). La lésion cutanée est caractérisée par une macule érythémateuse annulaire de plus de $5 \mathrm{~cm}$ de diamètre qui s'étend lentement de façon concentrique; elle est généralement indolore et non prurigineuse. La lésion s'éclaircit parfois en son centre, mais elle peut être plus uniformément érythémateuse $(19,23,24)$. II est important de noter que de nombreuses personnes peuvent réagir à la salive de tique et présenter une réaction localisée qui ressemble à un érythème migrant. Toutefois, contrairement à l'érythème migrant, cette réaction apparaît souvent dans les trois premiers jours de la morsure, mesure $5 \mathrm{~cm}$ de diamètre au maximum et ne s'étend pas.

L'érythème migrant peut être discret, voire passer inaperçu, en particulier si la morsure se trouve dans un endroit difficile à voir par le patient, derrière le genou, par exemple. Les épreuves diagnostiques ne peuvent pas détecter l'infection à ce stade et ne sont pas recommandées chez les patients en lien avec des régions d'endémie (6).

Traitement : Plusieurs essais cliniques menés aux États-Unis (23,25-28) et en Europe (29-31) ont éclairé le traitement antimicrobien de la maladie de Lyme au stade précoce. Dans ces essais, des patients ayant reçu un diagnostic clinique d'érythème migrant ont été répartis de façon aléatoire pour recevoir l'une de plusieurs options de traitement. Les agents utilisés comprenaient l'amoxicilline, la pénicilline, la ceftriaxone, un macrolide (érythromycine ou azithromycine) et une tétracycline ou la doxycycline à diverses doses et pour des durées diverses. L'azithromycine a été administrée pendant 5 ou 10 jours, les bêta-lactamines pendant 10 à 30 jours et la tétracycline/doxycycline pendant 10, 14 ou 20 à 21 jours. Un suivi, souvent d'une durée de plusieurs mois, a été effectué après la fin du traitement, à la recherche d'une résolution complète ou partielle des symptômes, de l'échec du traitement et de la présence de manifestations tardives de la maladie, jugées mineures ou majeures.

Selon les résultats de ces études, l'amoxicilline, l'azithromycine, la céfuroxime et la doxycycline offraient une efficacité comparable, indépendamment de la dose et de la durée du traitement. L'unique essai qui évaluait l'érythromycine a montré que cet agent était moins efficace que la pénicilline et la tétracycline. Une vaste étude de cohorte rétrospective incluant 607 patients a confirmé ces résultats, aucun échec au traitement n'ayant été constaté chez $99 \%$ des patients atteints d'une maladie de Lyme au stade précoce, localisée ou disséminée, deux ans après la fin du traitement, indépendamment de la durée de ce traitement : $\leq 10$ jours, 11 à 15 jours ou $>15$ jours (32). Une étude axée sur le stade disséminé précoce de la maladie de Lyme a comparé la ceftriaxone, 2 g, 1 f.p.j. pendant 2 semaines à la doxycycline, $100 \mathrm{mg} 2$ f.p.j. pendant 3 semaines (33). Les taux de guérison clinique ont été comparables pour les deux médicaments (85\% pour la ceftriaxone et $88 \%$ pour la doxycycline), $14 \%$ (doxycycline) et $27 \%$ (ceftriaxone) des patients ayant présenté des symptômes résiduels, généralement sous la forme de douleurs articulaires légères et de fatigue, neuf mois après la fin du traitement.

Pronostic : Les essais cliniques $(23,25-31)$ et les études d'observation $(32,34-36)$ fournissent des renseignements sur le pronostic de la maladie de Lyme au stade précoce chez les patients traités. Les thérapies actuellement recommandées permettent, au suivi à long terme, de constater une résolution complète des symptômes chez plus de 80 \% des patients. Bien que la présence de symptômes, comme la fatigue et les douleurs articulaires, ne soit pas rare après un traitement contre la maladie de Lyme, dans la plupart des études, le taux observé de manifestations tardives de la maladie au stade disséminé est nul ou faible $(<5 \%)$.

Afin de déterminer si les patients atteints de la maladie de Lyme au stade précoce étaient plus susceptibles d'avoir des symptômes persistants après une infection aiguë, en Slovénie, des chercheurs ont comparé une 
cohorte de patients traités pour un érythème migrant à un groupe témoin non atteint de la maladie de Lyme (36). Ils ont constaté que la fréquence de survenue de nouveaux symptômes ou d'aggravation des symptômes chez les patients atteints de la maladie n'était pas supérieure à celle observée dans le groupe témoin. À 12 mois, seulement $2,2 \%$ des patients atteints de la maladie ont signalé l'apparition de nouveaux symptômes ou une aggravation de leurs symptômes, aucun des patients ne présentant des symptômes invalidants. Ces résultats sont comparables à ceux d'une étude menée aux États-Unis chez des patients atteints et des patients exempts de la maladie de Lyme de 1984 à 1991 (37). La fréquence de survenue de nouveaux symptômes et la fréquence d'augmentation des difficultés dans l'exercice des activités de la vie quotidienne ont été comparables dans les deux groupes (patients atteints de la maladie de Lyme et sujets témoins appariés suivant l'âge exempts de la maladie).

En résumé, les données provenant de ces études donnent à penser que bien que les patients atteints de la maladie de Lyme puissent avoir des symptômes persistants ou de nouveaux symptômes une fois le traitement terminé, la fréquence de ces symptômes est comparable à celle observée chez des personnes exemptes de la maladie.

\section{Stade disséminé précoce de la maladie de Lyme (<3 mois)}

Le stade précoce peut être suivi d'un stade disséminé caractérisé par l'apparition de plusieurs lésions concentriques secondaires et de manifestations intermittentes concernant plusieurs systèmes : cardiaque, neurologique, oculaire ou articulaire. L'apparition de plusieurs lésions concentriques secondaires et de symptômes systémiques, notamment fièvre, douleurs articulaires, céphalée et adénopathie, se produit généralement plusieurs semaines après l'apparition de l'érythème migrant localisé (38). Dans une étude préliminaire, la moitié des patients atteints de la maladie ont fini par présenter plusieurs lésions d'érythème migrant (38).

Les autres manifestations caractéristiques du stade disséminé précoce de l'infection, telles que la cardite et l'infection neurologique de Lyme (ou neuroborréliose), ont tendance à survenir des semaines, voire des mois, après l'infection initiale (38). Les patients atteints de cardite de Lyme peuvent présenter des troubles de la conduction, des tachyarythmies, une myopéricardite ou une dysfonction myocardique (39). La manifestation la plus courante est le bloc atrio-ventriculaire (blocage cardiaque de grade 1 à 3), qui nécessite souvent une stimulation cardiaque temporaire. En général, la cardite survient dans les deux mois suivant l'apparition de l'érythème migrant, quoiqu'il n'existe parfois aucun antécédent de lésion.

Le traitement est efficace et la cardite est rarement mortelle (40). Bien que des données antérieures montrent que l'apparition d'une cardite survient chez jusqu'à $10 \%$ des patients atteints de maladie de Lyme n'ayant reçu aucun traitement (39), selon de récentes données, ce taux serait nettement plus bas; ceci peut être dû à une meilleure prise en compte de l'infection à Borrelia et à l'efficacité de son traitement (19). Des données recueillies aux États-Unis entre 1996 et 2006 donnent à penser que seuls 0,8\% des patients déclarés comme étant atteints de la maladie de Lyme présentent des troubles de la conduction (41). Les médecins doivent envisager la possibilité d'une maladie de Lyme lorsque des patients s'étant récemment rendus dans une région où la maladie est endémique présentent des troubles de la conduction inexpliqués. Toutefois, en l'absence d'antécédents d'érythème migrant, le tableau clinique n'est pas suffisamment spécifique pour justifier l'administration d'un traitement empirique sans confirmation sérologique (19).

Le spectre clinique de la neuroborréliose peut comprendre la méningite, la paralysie faciale, la radiculopathie avec atteinte motrice ou sensorielle et les symptômes cognitifs. En Amérique du Nord, la neuroborréliose est souvent caractérisée par une atteinte des nerfs crâniens (en particulier une paralyse de Bell) avec ou sans méningite à liquide clair. Les symptômes peuvent se manifester entre deux à huit semaines après l'apparition de l'érythème migrant (42). La maladie de Lyme peut provoquer une paralysie de Bell chez 10 à $50 \%$ des enfants et des adultes dans les régions d'endémie. La paralysie peut être bilatérale chez jusqu'à $23 \%$ des patients (4347). Les cliniciens doivent soupçonner la responsabilité d'une maladie de Lyme en présence d'une paralysie faciale observée chez un patient durant la saison des tiques dans les régions où la maladie est endémique, qui est accompagnée de maux de tête et d'autres signes neurologiques ne concernant pas les nerfs crâniens, notamment un œdème papillaire, ou est bilatérale (44,47-50). 
Dans les régions où la maladie de Lyme est endémique, il peut être difficile de différencier une neuroborréliose au stade précoce d'autres causes possibles d'infection du système nerveux central. En général, le liquide céphalorachidien est anormal, le nombre des leucocytes étant élevé à des taux comparables à ceux observés chez les patients atteints d'une méningite virale $(48,51)$. Toutefois, comparée à d'autres causes de méningite à liquide clair, la méningite de Lyme est moins susceptible d'être accompagnée de fièvre et plus susceptible d'être associée à une forte prépondérance de cellules mononucléaires (pourcentage total de monocytes et de lymphocytes) dans le liquide céphalorachidien, à des maux de tête prolongés et à une atteinte des nerfs crâniens chez les patients concernés $(48,52,53)$.

La règle des sept : Une règle de prévision clinique a été établie afin de faciliter la stratification de la probabilité de la présence d'une méningite de Lyme chez les enfants. La présence d'une méningite de Lyme est peu probable chez les enfants qui répondent à la totalité des critères suivants : maux de tête durant moins de sept jours, taux de cellules mononucléaires inférieur à $70 \%$ relevé dans le liquide céphalorachidien, et absence de paralysie du septième nerf crânien. Cette « règle des 7 » permet de différencier la méningite à liquide claire de la neuroborréliose de Lyme avec une sensibilité de $96 \%$ et une spécificité de $95 \%$. Selon le principe de cette règle, les patients qui ne répondent pas à ces critères peuvent être traités de façon conservative en attendant les résultats des tests sérologiques de dépistage de la maladie de Lyme $(53,54)$.

Traitement : Une étude menée aux États-Unis au début des années 1990 auprès de patients atteints d'une maladie de Lyme au stade disséminé (sans méningite) a comparé la ceftriaxone, $2 \mathrm{~g} 1$ f.p.j. pendant 14 jours, à la doxycycline, $100 \mathrm{mg} 2$ f.p.j. pendant 21 jours (33). Le taux de guérison était semblable pour les deux traitements (88\% pour la ceftriaxone et $85 \%$ pour la doxycycline), $27 \%$ des patients traités par la ceftriaxone et $14 \%$ de ceux traités par la doxycycline ayant présenté des symptômes résiduels à la fin du traitement.

Dans une vaste étude de série de cas incluant 101 adultes atteints d'une paralysie de Bell liée à la maladie de Lyme, une résolution complète de la paralysie a été constatée chez plus de $86 \%$ des patients, dans un délai médian de 26 jours (fourchette allant de 1 à 270 jours) malgré le fait que seuls $36 \%$ avaient reçu une antibiothérapie. Quinze pour cent présentaient une légère faiblesse résiduelle et seul un des patients présentait un déficit grave. Les patients présentant une dysfonction résiduelle étaient plus susceptibles d'être atteints d'une maladie bilatérale (43).

Le pronostic chez les enfants est tout aussi favorable. Dans une étude de cohorte, $94 \%$ des enfants traités par des antibiotiques étaient asymptomatiques quatre semaines après la fin du traitement. Tous les patients sauf un ont connu une résolution de leurs symptômes dans les six mois et deux ans plus tard, aucun des enfants ne présentait de signes de maladie chronique (34). La plupart de 43 enfants ayant présenté une paralysie de Bell attribuable à la maladie de Lyme se sont estimés guéris et aucune différence n'a été observée entre les enfants infectés et non infectés sur le plan des activités quotidiennes (55). Ces études menées chez des patients atteints de neuroborréliose de Lyme soulignent combien une prise en compte et un traitement précoces sont importants pour améliorer la réponse au traitement. Parallèlement, il a été démontré qu'un traitement prolongé par l'amoxicilline administré par voie orale après l'administration d'une cure de trois semaines de ceftriaxone par voie intraveineuse n'avait pas d'effet sur le pronostic par rapport au placebo (56).

\section{Stade tardif de la maladie de Lyme ( $>3$ mois)}

En l'absence de traitement, la maladie de Lyme peut évoluer en une maladie au stade tardif, caractérisée par la présence de signes persistants d'arthrite ou de neuroborréliose. Les souches différentes de Borrelia présentes en Amérique du Nord et en Europe sont associées à des tableaux cliniques qui sont distincts, mais se recoupent. En Europe, le stade tardif de la maladie est caractérisé par des syndromes dermatologiques qui sont rares en Amérique du Nord, notamment l'acrodermatite chronique atrophiante et le lymphocytome cutané bénin (24). En Amérique du Nord, l'arthrite est une manifestation beaucoup plus courante de la maladie de Lyme au stade tardif, alors qu'en Europe la neuroborréliose est plus répandue et se manifeste sous la forme d'une encéphalite chronique ou d'une radiculopathie douloureuse appelée syndrome de Bannwarth dans jusqu'à $86 \%$ des cas (57).

En cas de suspicion d'une maladie de Lyme européenne fondée sur le tableau clinique et les antécédents de voyage du patient, le laboratoire doit en être avisé pour s'assurer qu'il utilise les épreuves sérologiques appropriées, les épreuves de confirmation n'étant pas les mêmes pour les borrélies d'Amérique du Nord et 
celles d'Europe. Une comparaison plus détaillée des différences entre la maladie de Lyme d'Amérique du Nord et celle d'Europe, est fournie par Hengge et ses collègues (24).

\section{Arthrite de la maladie de Lyme au stade tardif}

En Amérique du Nord, jusqu'à $60 \%$ des cas de maladie de Lyme non traités présentent une mono-arthrite ou une oligo-arthrite, touchant généralement les genoux, et, dans une moindre mesure, d'autres articulations, notamment celles de la hanche, du coude et du poignet (58-60 ans). Dans une étude, tous les patients atteints d'une arthrite liée au stade tardif de la maladie de Lyme ont vu les symptômes apparaître dans les deux ans de la contraction de l'infection. Sur 16 patients n'ayant pas reçu de traitement pour une paralysie du nerf facial liée à la maladie de Lyme, 14 ont ultérieurement contracté une arthrite (61). Le souvenir d'avoir subi la morsure d'une tique ne s'est pas avéré utile à la pose du diagnostic d'arthrite de Lyme, car jusqu'à $84 \%$ des cas ne se souvenaient pas d'avoir été exposés à l'animal $(32,62)$.

La sérologie de la maladie de Lyme est très sensible et spécifique vis-à-vis du diagnostic d'arthrite de Lyme et un résultat négatif permet essentiellement d'écarter l'existence d'une infection (6). Cependant, les résultats de dépistage de la maladie de Lyme sont parfois longs à obtenir et étant donné que le tableau clinique de l'arthrite de Lyme peut ressembler à celui de l'arthrite purulente qui impose l'instauration immédiate d'un traitement, il est important d'essayer de différencier ces deux pathologies.

Trois études ont tenté d'élaborer un modèle de prédiction clinique afin de distinguer l'arthrite de Lyme de l'arthrite purulente dans les régions où la maladie de Lyme est endémique Bien que les deux pathologies aient des caractéristiques qui se recoupent, l'arthrite de Lyme tend à toucher surtout les genoux, est plus rarement accompagnée de fièvre et de signes locaux d'inflammation et est généralement caractérisée par un taux moins élevé de marqueurs de l'inflammation (62-64). Une étude a constaté que le refus de mise en charge était le facteur de prédiction le plus puissant qui permette de distinguer l'arthrite purulente de la maladie de Lyme (63). Une autre étude menée chez des enfants a constaté qu'une vitesse de sédimentation inférieure à $40 \mathrm{~mm} / \mathrm{heure}$ et un compte de leucocytes inférieur à $10 \times 10^{3}$ cellules $/ \mathrm{mm}^{3}$ dans le sang périphérique permettraient efficacement d'écarter la présence d'une arthrite purulente (64). Malgré le liquide synovial inflammatoire et la numération leucocytaire élevée relevés dans l'arthrite purulente, des recoupements existent avec l'arthrite de Lyme, la numération cellulaire pouvant varier de $1700 \times 10^{3}$ cellules/ $\mu \mathrm{L}$ à plus de $100000 \times 10^{3}$ cellules/ $\mu \mathrm{L}$, avec prédominance de cellules polymorphonucléaires $(58,60,63)$. Dans une étude, le taux des marqueurs inflammatoires était plus élevé chez les enfants atteints de la maladie de Lyme que chez les adultes (60).

Jusqu'à $10 \%$ des patients peuvent présenter des signes de synovite six mois après la fin du traitement contre l'arthrite de Lyme, pathologie nommée « arthrite de Lyme réfractaire aux antibiotiques » (65). À l'heure actuelle, le recours à la réaction en chaîne de la polymérase (PCR) pour détecter l'ADN de Borrelia dans le diagnostic de la maladie de Lyme est recommandé uniquement chez les patients présentant un œdème synovial persistant dans le but de déterminer la nécessité d'une reprise de traitement (19). L'interprétation d'un résultat positif après un traitement primaire est toutefois difficile. Certaines données semblent indiquer que les Borrelia détectées ne sont pas toutes viables, ce qui laisse un doute quant à la présence effective d'une infection persistante chez ces patients (66).

Traitement de l'arthrite liée à la maladie de Lyme au stade tardif : Peu d'études se sont penchées sur le traitement de l'arthrite liée au stade tardif de la maladie de Lyme. Deux études $(67,68)$ comparant la pénicilline ou la ceftriaxone à un placebo chez des adultes atteints d'une arthrite liée au stade tardif de la maladie de Lyme ont révélé que l'antibiothérapie était bénéfique; bien que le taux de guérison, plus élevé avec la ceftriaxone qu'avec la pénicilline, ne dépassait pas les $50 \%$. Dans un essai européen à répartition aléatoire comparant la pénicilline et la ceftriaxone administrées toutes deux par voie intraveineuse, pendant 10 jours, chez des patients âgés de 13 ans et plus, le taux de rémission des symptômes a été de 87,9\% avec la ceftriaxone et de 61,3\% avec la pénicilline. Enfin, selon une étude menée vers le milieu des années 2000 aux États-Unis chez des enfants et des adultes atteints d'une maladie de Lyme au stade tardif (69), un traitement de 28 jours (taux de guérison de $70 \%$ ) par la ceftriaxone ne présentait aucun bénéfice par rapport à un traitement de14 jours (taux de guérison de $76 \%$ ) sur le plan de la guérison clinique et était associé à un plus grand nombre d'abandons du traitement pour cause d'effets indésirables. Dans certaines séries de cas, jusqu'à $25 \%$ des enfants et $50 \%$ des adultes ont dû recevoir une deuxième cure d'antibiotiques en raison de la présence de symptômes d'arthrite réfractaire. Les cas d'arthrite réfractaire ont pu être traités avec succès par des anti-inflammatoires non 
stéroïdiens, des injections de stéroïdes ou un traitement de fond. Aucune séquelle à long terme n'a été observée $(59,60)$.

\section{Neuroborréliose au stade tardif}

En Amérique du Nord, la neuroborréliose se traduit le plus souvent par une encéphalopathie subaiguë accompagnée de troubles cognitifs mineurs, alors qu'en Europe, l'encéphalomyélite chronique caractérisée par une paraparésie spastique et un déficit cognitif est plus courante $(24,70)$. Dans une étude de série de cas incluant 37 patients américains, la neuroborréliose s'est manifestée environ deux ans après l'apparition de l'érythème migrant sous la forme d'une encéphalopathie subaiguë, d'une polyradiculoneuropathie axonale accompagnée d'anomalies sensorielles ou électromyographiques objectives ou d'une leucoencéphalite, ou encore d'une combinaison de ces trois pathologies (42). II est important de souligner la nature objective des constatations établies et des signes sérologiques d'infection concernant ces patients.

Traitement de la neuroborréliose au stade tardif : Toutes les études primaires portant sur le traitement de la neuroborréliose relevées lors de notre recherche ont été entreprises en Europe (56,71-77). Bien que les manifestations cliniques de la maladie de Lyme diffèrent sensiblement entre les États-Unis et l'Europe, les principes de traitement sont présumément les mêmes. Ces différentes études ont comparé un éventail varié de médicaments, de doses et de durée de traitement différents. En général, toutefois, les comparateurs utilisés étaient la ceftriaxone/céfotaxime par voie intraveineuse, la pénicilline par voie intraveineuse ou la doxycycline par voie orale (200 à 400 mg par jour) pour des durées allant de 10 jours à trois semaines. Les patients étaient atteints d'une maladie neurologique au stade précoce ou tardif et le suivi était souvent assuré pendant plusieurs mois après l'arrêt du traitement. L'efficacité du traitement était comparable dans les différents groupes de traitement, mais la dose de doxycycline était plus élevée dans certaines de ces études que celle indiquée dans le traitement de la maladie de Lyme au stade précoce. En général, le taux de réussite du traitement dépassait les $80 \%$. Un taux de $33 \%$ a toutefois été relevé dans un groupe de patients atteints d'une neuroborréliose de Lyme au stade tardif traités par la ceftriaxone pendant deux semaines.

\section{Quel est le pronostic en cas de traitement différé?}

II existe relativement peu de données sur les effets d'un traitement différé. Deux études rétrospectives ont montré que les patients ayant présenté des symptômes pendant une durée prolongée avant de recevoir un traitement étaient plus susceptibles de présenter des symptômes musculosquelettiques et cognitifs subjectifs persistants. Soulignons toutefois l'absence de constatations physiques et de signes d'anomalie neurocognitive objectivement établis par rapport à des témoins non infectés $(78,79)$. Des résultats semblables ont été observés chez des patients ayant participé à des études portant sur la maladie de Lyme au stade précoce. Selon ces résultats, les patients n'ayant pas reçu de traitement à l'apparition initiale d'une paralysie faciale étaient plus susceptibles d'avoir des douleurs et des limitations physiques et d'obtenir un score composite physique plus faible sur l'échelle SF-36 (questionnaire abrégé en 36 points d'auto-évaluation de l'état de santé) que les témoins traités par antibiotiques à l'apparition de la paralysie. Ils étaient également plus susceptibles d'avoir des symptômes neurocognitifs bénins, bien que leur score composite mental (questionnaire SF-36 normalisé) n'était pas différent de celui du groupe témoin (61).

\section{Les patients atteints de la maladie de Lyme peuvent-ils avoir des symptômes persistants après le traitement?}

À l'évidence, une proportion de patients chez lesquels la présence d'une maladie de Lyme antérieure a été confirmée continuent d'avoir des symptômes après un traitement classique par les antimicrobiens. Les expressions «maladie de Lyme chronique » et «syndrome post-Lyme » ont été utilisées par certains cliniciens pour décrire les cas de certains patients présentant des symptômes persistants plus de six mois après un traitement par des agents recommandés. Les symptômes persistants comprennent la fatigue, des douleurs musculosquelettiques généralisées et un déficit cognitif, non étayés par des constatations ou des signes microbiologiques d'infection active objectivement établis.

Vu l'existence, chez certains patients, de symptômes persistants après le traitement de la maladie de Lyme, la question de l'intérêt d'un traitement prolongé par les antimicrobiens s'est posée. Quatre essais à répartition aléatoire et contrôlés par placebo, tous menés aux États-Unis, se sont penchés sur cette question. La plus 
grande de ces études (80) regroupait deux études distinctes (études de cas séropositifs et séronégatifs) menées chez des patients ayant fait l'objet d'un diagnostic clinique de maladie de Lyme aiguë. Les patients évalués dans le cadre de ces deux études avaient été antérieurement traités selon un protocole d'antibiothérapie recommandé et présentaient toujours des symptômes. Les sujets ont été admis dans l'étude de cas séropositifs ou séronégatifs en fonction de leur statut sérologique au moment de l'inscription. Un total de 129 patients a été inscrit dans les deux études, et les participants ont été répartis de façon aléatoire pour recevoir soit la ceftriaxone, $2 \mathrm{~g} 1$ f.p.j. pendant 30 jours, suivie de l'administration de doxycycline pendant 60 jours, soit un placebo. Les critères d'évaluation comprenaient la cognition, la mémoire, la douleur et les activités de la vie quotidienne.

Aucune différence sur le plan des résultats n'a été constatée entre les groupes de traitement et les groupes placebo, ni dans l'étude des cas séronégatifs ni dans celle des cas séropositifs. Les essais ont été interrompus prématurément après la réalisation d'une analyse intermédiaire par le comité de surveillance et de suivi indiquant qu'ils ne permettraient pas d'établir de différence entre le traitement et le placebo. Une étude secondaire fondée sur les données de cet essai n'a également constaté aucune différence entre les groupes de traitement et les groupes témoins sur le plan de l'humeur et de la qualité de vie liée aux fonctions cognitives (81).

Krupp et ses collègues ont comparé la ceftriaxone administrée à raison de $2 \mathrm{~g}$ par jour pendant 28 jours à un placebo chez 55 patients dont la maladie de Lyme avait été traitée antérieurement selon un protocole classique (82). Bien qu'une diminution de la fatigue ait été constatée dans le groupe recevant la ceftriaxone, les résultats d'essais formels d'évaluation des fonctions cognitives n'ont révélé aucune différence entre les groupes. Parmi les patients, on a relevé trois cas d'infection due à la ligne de perfusion et un cas de réaction anaphylactique à la ceftriaxone. Enfin, 37 adultes ont été répartis de façon aléatoire pour recevoir soit la ceftriaxone soit un placebo pendant 10 semaines après la fin d'un traitement contre la maladie de Lyme (83). Chez les patients ayant reçu la ceftriaxone, de légères améliorations non soutenues des fonctions cognitives ont été constatées, accompagnées d'un taux d'effets indésirables de $26,1 \%$, ce taux étant de $7,1 \%$ dans le groupe placebo.

À la lumière de ces résultats, les lignes directrices de la Infectious Diseases Society of America ne recommandent pas l'instauration d'un traitement additionnel ou prolongé par les antimicrobiens chez les patients ayant suivi un traitement classique contre la maladie de Lyme. (19). Les bénéfices éventuels d'un tel traitement sont minimes et non soutenus et ont été associés à un risque excessif d'effets indésirables potentiellement mortels.

\section{Comment la maladie de Lyme doit-elle être prise en charge durant la grossesse?}

Bien que des cas de maladie de Lyme chez des femmes enceintes et des cas sporadiques de transmission transplacentaire de $B$. burgdorferi aient été signalés, aucun lien n'a été éclairement établi entre l'infection fœtale et les issues de grossesse défavorables $(84,85)$. Les études cliniques, sérologiques et épidémiologiques portant sur l'infection à Borrelia durant la grossesse n'ont pas permis de montrer l'existence d'un lien entre l'infection et les issues de grossesses défavorables $(84-86)$. Étant donné que la doxycycline est contre-indiquée durant la grossesse, les options thérapeutiques et prophylactiques ne sont pas les mêmes pour les femmes enceintes que pour les autres adultes.

Certaines données donnent à penser que l'administration d'ampicilline pendant 10 jours peut être une stratégie prophylactique efficace, bien que le risque d'éruption cutanée en réaction aux bêta-lactamines soit supérieur au risque de contracter la maladie de Lyme. Les lignes directrices de I'IDSA (Infectious Diseases Society of America) ne recommandent donc pas l'administration d'une prophylaxie durant la grossesse $(18,19,86)$. Lorsqu'une femme enceinte subit la morsure d'une tique Ixodes, il est recommandé de surveiller pendant 30 jours l'apparition éventuelle des signes et symptômes de la maladie de Lyme et d'administrer à la patiente un traitement par l'amoxicilline ou la céfuroxime si elle contracte la maladie (19).

\section{Une réinfection par Borrelia burgdorferi est-elle possible?}

La rechute est une récurrence de symptômes qui survient lorsque le traitement n'est pas parvenu à guérir l'infection initiale alors que la réinfection est une récurrence de symptômes due à une nouvelle exposition à une tique infectée qui provoque une nouvelle infection. Bien que la rechute d'un érythème migrant soit possible en 
l'absence d'antibiothérapie (23), il est probable que sa récurrence après un traitement réussi soit due à une réinfection plutôt qu'à une rechute (87).

Une réinfection peut se produire chez 2 à $21 \%$ des patients vivant dans des régions d'endémie ayant des antécédents de maladie de Lyme (78,88 - 90). Une réinfection est généralement caractérisée par l'apparition d'un érythème migrant à un endroit différent de la lésion initiale, plus de 1 à 2,5 ans après l'infection initiale, jamais dans les 11 mois suivant la première infection $(87,89,91)$. Dans une série de cas, la réinfection s'est traduite par l'apparition d'un érythème migrant à un site différent de celui de l'infection précédente chez $79 \%$ des patients et par une maladie fébrile accompagnée de myalgies chez $21 \%$ d'entre eux. La réinfection après une maladie de Lyme au stade tardif caractérisée par une arthrite ou une neuroborréliose est très rare $(90,91)$.

Le diagnostic en laboratoire de la réinfection pose un véritable défi car la sérologie, y compris le dosage des IgM, peut rester positive pendant de nombreuses années (92). Le diagnostic repose sur la présence constatée d'un nouvel érythème migrant à un site différent de la lésion initiale. Des données récentes donnent à penser que le pronostic des patients traités pour une réinfection est excellent. Les symptômes de fatigue signalés étaient moins fréquents chez les patients ayant subi une réinfection que dans le groupe témoin non infecté et que chez les patients infectés pour la première fois, ce qui permet de penser que le risque de symptômes persistants est moins élevé lors d'une réinfection (91).

\section{Conclusions}

La maladie de Lyme est une infection émergente au Canada, et il est important que les cliniciens en connaissent l'épidémiologie, le tableau clinique et la prise charge. Bien que l'apparition de cas sporadiques de maladie de Lyme transmis par des tiques infectées transportées par des oiseaux migrateurs soit possible, les personnes qui vivent ou voyagent dans des régions où la maladie est endémique sont davantage exposées au risque d'infection. Un relevé précis des antécédents de voyage est essentiel lorsqu'une personne présente des symptômes évocateurs de la maladie de Lyme, en particulier si ces symptômes correspondent à l'un des syndromes neurologiques plus couramment rencontrés en Europe. Il est important d'informer le laboratoire de tout antécédent de voyage pertinent, car les épreuves sérologiques ne sont pas les mêmes pour les souches de Borrelia d'Amérique du Nord et d'Europe.

La population de tiques étant en constante expansion, il peut être difficile de définir clairement les régions où le risque d'exposition à la maladie de Lyme est le plus élevé, et il est important de se tenir au courant de l'épidémiologie locale de la maladie auprès des autorités locales de la santé publique.

\section{Conflit d'intérêts}

II n'y a aucun conflit d'intérêts à déclarer. 


\section{Références}

(1) Kuehn BM. CDC estimates 300000 US cases of Lyme disease annually. JAMA. 2013; 310:1110.

(2) Henry B, Roth D, Reilly R, MacDougall L, Mak S, Li M, et al. How big is the Lyme problem? Using novel methods to estimate the true number of Lyme disease cases in British Columbia residents from 1997 to 2008. Vector Borne Zoonotic Dis. 2011; 11:863-8.

(3) Owens B. Risk of tick-borne infections on the rise. CMAJ. 2013; 185:E711.

(4) Ogden, N.H., Koffi, J.K., Pelcat, Y., Lindsay, L.R. Risque environnemental pour la maladie de Lyme dans l'est et le centre du Canada : un sommaire d'informations récentes en matière de surveillance. Relevé des maladies transmissibles au Canada, vol. 40, p. 74-83, 2014.

(5) Ogden NH, Lindsay LR, Morshed M, Sockett PN, Artsob H. The emergence of Lyme disease in Canada. CMAJ. 2009; 180:1221-4.

(6) Lindsay LR, Bernat K, Dibernardo A. Laboratory diagnostics for Lyme disease. CCDR 2014; 40:232-241

(7) Huegli D, Moret J, Rais O, Moosmann Y, Erard P, Malinverni R, et al. Prospective study on the incidence of infection by Borrelia burgdorferi sensu lato after a tick bite in a highly endemic area of Switzerland. Ticks Tick Borne Dis. 2011; 2:129-36.

(8) Costello CM, Steere AC, Pinkerton RE, Feder HM Jr. A prospective study of tick bites in an endemic area for Lyme disease. J Infect Dis. 1989; 159:136-9.

(9) Shapiro ED, Gerber MA, Holabird NB, Berg AT, Feder HM Jr, Bell GL, et al. A controlled trial of antimicrobial prophylaxis for Lyme disease after deer-tick bites. N Engl J Med 1992; 327:1769-74.

(10) Agre F, Schwartz R. The value of early treatment of tick bites for the prevention of Lyme disease. Am J Dis Child. 1993; 147:945-7.

(11) Nadelman RB, Nowakowski J, Fish D, Falco RC, Freeman K, McKenna D, et al. Prophylaxis with single-dose doxycycline for the prevention of Lyme disease after an Ixodes scapularis tick bite. N Engl J Med. 2001; 345:79-84.

(12) Sood SK, Salzman MB, Johnson BJB, Happ CM, Feig K, Carmody L, et al. Duration of tick attachment as a predictor of the risk of Lyme disease in an area in which Lyme disease is endemic. J Infect Dis. 1997; 175:996-9.

(13) Pal U, Yang X, Chen M, Bockenstedt LK, Anderson JF, Flavell RA et al. OspC facilitates Borrelia burgdorferi invasion of Ixodes scapularis salivary glands. J Clin Invest. 2004; 113:220-30.

(14) Piesman J, Zeidner NS, Schneider BS. Dynamic changes in Borrelia burgdorferi populations in Ixodes scapularis (Acari: Ixodidae) during transmission: studies at the mRNA level. Vector Borne Zoonotic Dis. 2003; 3:125-32.

(15) Schwan TG, Piesman J. Temporal changes in outer surface proteins A and C of the lyme disease-associated spirochete, Borrelia burgdorferi, during the chain of infection in ticks and mice. J Clin Microbiol. 2000; 38:382-8.

(16) Radolf JD, Caimano MJ, Stevenson B, Hu LT. Of ticks, mice and men: understanding the dual-host lifestyle of Lyme disease spirochaetes. Nat Rev Microbiol. 2012; 10:87-99.

(17) Warshafsky S, Nowakowski J, Nadelman RB, Kamer RS, Peterson SJ, Wormser GP. Efficacy of antibiotic prophylaxis for prevention of Lyme disease. J Gen Intern Med. 1996; 11:329-33. 
(18) Warshafsky S, Lee DH, Francois LK, Nowakowski J, Nadelman RB, Wormser GP. Efficacy of antibiotic prophylaxis for the prevention of Lyme disease: an updated systematic review and meta-analysis. J Antimicrob Chemother. 2010; 65:1137-44.

(19) Wormser GP, Dattwyler RJ, Shapiro ED, Halperin JJ, Steere AC, Klempner MS, et al. The clinical assessment, treatment, and prevention of Lyme disease, human granulocytic anaplasmosis, and babesiosis: clinical practice guidelines by the Infectious Diseases Society of America. Clin Infect Dis. Nov 1 2006; 43:1089-134.

(20) Steere AC, Sikand VK, Schoen RT, Nowakowski J. Asymptomatic infection with Borrelia burgdorferi. Clin Infect Dis. 2003; 37:528-32.

(21) Petersen LR, Sweeney AH, Checko PJ, Magnarelli LA, Mshar PA, Gunn RA, et al. Epidemiological and clinical features of 1,149 persons with Lyme disease identified by laboratory-based surveillance in Connecticut. Yale $\mathrm{J}$ of Biol Med 1989; 62:253-62.

(22) Steere AC, Sikand VK. The presenting manifestations of Lyme disease and the outcomes of treatment. N Engl J Med. 2003; 348:2472-74.

(23) Steere AC, Bartenhagen NH, Craft JE, Hutchinson GJ, Newman JH, Rahn DW, et al. The early clinical manifestations of Lyme disease. Ann Intern Med. 1983; 99:76-82.

(24) Hengge UR, Tannapfel A, Tyring SK, Erbel R, Arendt G, Ruzicka T. Lyme borreliosis. Lancet Infect Dis. 2003; 3:489-500.

(25) Nadelman RB, Luger SW, Frank E, Wisniewski M, Collins JJ, Wormser GP. Comparison of cefuroxime axetil and doxycycline in the treatment of early Lyme disease. Ann Intern Med. 1992; 117:273-80.

(26) Luger SW, Paparone P, Wormser GP, Nadelman RB, Grunwaldt E, Gomez G, et al. Comparison of cefuroxime axetil and doxycycline in treatment of patients with early Lyme disease associated with erythema migrans. Antimicrob Agents Chemother. 1995; 39:661-7.

(27) Luft BJ, Dattwyler RJ, Johnson RC, Luger SW, Bosler EM, Rahn DW, et al. Azithromycin compared with amoxicillin in the treatment of erythema migrans. A double-blind, randomized, controlled trial. Ann Intern Med. 1996; 124:785-91.

(28) Wormser GP, Ramanathan R, Nowakowski J, McKenna D, Holmgren D, Visintainer P, et al. Duration of antibiotic therapy for early Lyme disease. A randomized, double-blind, placebo-controlled trial. Ann Intern Med. 2003; 138:697-704.

(29) Strle F, Maraspin V, Furlan-Lotric S, Cimperman J. Epidemiological study of a cohort of adult patients with Erythema migrans registered in Slovenia in 1993. Eur J Epidemiol. 1996; 12:503-7.

(30) Weber K, Wilske B, Preac-Mursic V, Thurmayr R. Azithromycin versus penicillin V for the treatment of early Lyme borreliosis. Infection. 1993; 21:367-72.

(31) Barsic B, Maretic T, Majerus L, Strugar J. Comparison of azithromycin and doxycycline in the treatment of erythema migrans. Infection. 2000; 28:153-6.

(32) Kowalski TJ, Tata S, Berth W, Mathiason MA, Agger WA. Antibiotic treatment duration and long-term outcomes of patients with early lyme disease from a lyme disease-hyperendemic area. Clin Infect Dis. 2010; 50:512-20.

(33) Dattwyler RJ, Luft BJ, Kunkel MJ, Finkel MF, Wormser GP, Rush TJ, et al. Ceftriaxone compared with doxycycline for the treatment of acute disseminated Lyme disease. N Engl J Med. 1997; 337:289-94.

(34) Gerber MA, Shapiro ED, Burke GS, Parcells VJ, Bell GL. Lyme disease in children in southeastern Connecticut. Pediatric Lyme Disease Study Group. N Engl J Med. 1996; 335:1270-4. 
(35) Smith RP, Schoen RT, Rahn DW, Sikand VK, Nowakowski J, Parenti DL, et al. Clinical characteristics and treatment outcome of early Lyme disease in patients with microbiologically confirmed erythema migrans. Ann Intern Med. 2002; 136:421-8.

(36) Cerar D, Cerar T, Ruzić-Sabljić E, Wormser GP, Strle F. Subjective symptoms after treatment of early Lyme disease. Am J Med. 2010; 123:79-86.

(37) Seltzer EG, Gerber MA, Cartter ML, Freudigman K, Shapiro ED. Long-term outcomes of persons with Lyme disease. JAMA. 2000; 283:609-16.

(38) Steere AC. Lyme disease. N Engl J Med. 1989; 321:586-96.

(39) Sigal LH. Early disseminated Lyme disease: cardiac manifestations. Am J Med. 1995; 98:25S-28S.

(40) Centers for Disease Control and Prevention (CDC). Three sudden cardiac deaths associated with Lyme carditis - United States, November 2012-July 2013. MMWR Morb Mortal Wkly Rep. 2013; 62:993-6.

(41) Bacon RM, Kugeler KJ, Mead PS; Centers for Disease Control and Prevention (CDC). Surveillance for Lyme disease--United States, 1992-2006. MMWR Surveill Summ. 2008; 57:1-9.

(42) Logigian EL, Kaplan RF, Steere AC. Chronic neurologic manifestations of Lyme disease. N Engl J Med. 1990; 323:1438-44.

(43) Clark JR, Carlson RD, Sasaki CT, Pachner AR, Steere AC. Facial paralysis in Lyme disease. Laryngoscope. 1985; 95:1341-5.

(44) Kuiper H, Devriese PP, de Jongh BM, Vos K, Dankert J. Absence of Lyme borreliosis among patients with presumed Bell's palsy. Arch Neurol. 1992; 49:940-3.

(45) Halperin JJ, Golightly M. Lyme borreliosis in Bell's palsy. Long Island Neuroborreliosis Collaborative Study Group. Neurology. 1992; 42:1268-70.

(46) Cook SP, Macartney KK, Rose CD, Hunt PG, Eppes SC, Reilly JS. Lyme disease and seventh nerve paralysis in children. Am J Otolaryngol. 1997; 18:320-3.

(47) Nigrovic LE, Thompson AD, Fine AM, Kimia A. Clinical predictors of Lyme disease among children with a peripheral facial palsy at an emergency department in a Lyme disease-endemic area. Pediatrics. 2008; 122:e1080-5.

(48) Eppes SC, Nelson DK, Lewis LL, Klein JD. Characterization of Lyme meningitis and comparison with viral meningitis in children. Pediatrics. 1999; 103:957-60.

(49) Ljøstad U, Økstad S, Topstad T, Mygland A, Monstad P. Acute peripheral facial palsy in adults. J Neurol. 2005; 252:672-6.

(50) Bremell D, Hagberg L. Clinical characteristics and cerebrospinal fluid parameters in patients with peripheral facial palsy caused by Lyme neuroborreliosis compared with facial palsy of unknown origin (Bell's palsy). BMC Infect Dis. 2011; 11:215.

(51) Bremell D, Mattsson N, Wallin F, Henriksson J, Wall M, Blennow K, et al. Automated cerebrospinal fluid cell count - New reference ranges and evaluation of its clinical use in central nervous system infections. Clin Biochem. 2014; 47:25-30.

(52) Avery RA, Frank G, Glutting JJ, Eppes SC. Prediction of Lyme meningitis in children from a Lyme diseaseendemic region: a logistic-regression model using history, physical, and laboratory findings. Pediatrics. 2006; 117:e1-7.

(53) Cohn KA, Thompson AD, Shah SS, Hines EM, Lyons TW, Welsh EJ, et al. Validation of a clinical prediction rule to distinguish Lyme meningitis from aseptic meningitis. Pediatrics. 2012; 129:e46-53. 
(54) Garro AC, Rutman M, Simonsen K, Jaeger JL, Chapin K, Lockhart G. Prospective validation of a clinical prediction model for Lyme meningitis in children. Pediatrics. 2009; 123:e829-34.

(55) Vázquez M, Sparrow SS, Shapiro ED. Long-term neuropsychologic and health outcomes of children with facial nerve palsy attributable to Lyme disease. Pediatrics. 2003; 112:e93-7.

(56) Oksi J, Nikoskelainen J, Hiekkanen H, Lauhio A, Peltomaa M, Pitkäranta A, et al. Duration of antibiotic treatment in disseminated Lyme borreliosis: a double-blind, randomized, placebo-controlled, multicenter clinical study. Eur J Clin Microbiol Infect Dis. 2007; 8:571-81.

(57) Pachner AR, Steiner I. Lyme neuroborreliosis: infection, immunity and inflammation. Lancet Neurol. 2007; 6:544-552.

(58) Steere AC, Schoen RT, Taylor E. The clinical evolution of Lyme arthritis. Ann Intern Med. 1987; 107:725-31.

(59) Tory HO, Zurakowski D, Sundel RP. Outcomes of children treated for Lyme arthritis: results of a large pediatric cohort. J Rheumatol. 2010; 37:1049-55.

(60) Daikh BE, Emerson FE, Smith RP, Lucas FL, McCarthy CA. Lyme arthritis: a comparison of presentation, synovial fluid analysis, and treatment course in children and adults. Arthritis Care Res. 2013; 65:1986-90.

(61) Kalish RA, Kaplan RF, Taylor E, Jones-Woodward L, Workman K, Steere AC. Evaluation of study patients with Lyme disease, 10-20-year follow-up. J Infect Dis. 2001; 183:453-60.

(62) Thompson A, Mannix R, Bachur R. Acute pediatric monoarticular arthritis: distinguishing Lyme arthritis from other etiologies. Pediatrics. 2009; 123:959-65.

(63) Milewski MD, Cruz Al Jr, Miller CP, Peterson AT, Smith BG. Lyme arthritis in children presenting with joint effusions. J Bone Joint Surg Am. 2011; 93:252-60.

(64) Deanehan JK, Kimia AA, Tan Tanny SP, Milewski MD, Talusan PG, Smith BG, et al. Distinguishing Lyme from septic knee monoarthritis in Lyme disease-endemic areas. Pediatrics. 2013; 131:e695-701.

(65) Steere AC, Levin RE, Molloy PJ, Kalish RA, Abraham JH 3rd, Liu NY, et al. Treatment of Lyme arthritis. Arthritis Rheum. 1994; 37:878-88.

(66) Li X, McHugh GA, Damle N, Sikand VK, Glickstein L, Steere AC. Burden and viability of Borrelia burgdorferi in skin and joints of patients with erythema migrans or Lyme arthritis. Arthritis Rheum. 2011; 63:2238-47.

(67) Caperton EM, Heim-Duthoy KL, Matzke GR, Peterson PK, Johnson RC. Ceftriaxone therapy of chronic inflammatory arthritis. A double-blind placebo controlled trial. Arch Intern Med. 1990; 150:1677-82.

(68) Steere AC, Green J, Schoen RT, Taylor E, Hutchinson GJ, Rahn DW, et al. Successful parenteral penicillin therapy of established Lyme arthritis. N Engl J Med. 1985; 312:869-74.

(69) Dattwyler RJ, Wormser GP, Rush TJ, Finkel MF, Schoen RT, Grunwaldt E, et al. A comparison of two treatment regimens of ceftriaxone in late Lyme disease. Wien Klin Wochenschr. 2005; 117:393-7.

(70) Coyle PK, Schutzer SE. Neurologic aspects of Lyme disease. Med Clin North Am. 2002; 86:261-84.

(71) Pfister HW, Preac-Mursic V, Wilske B, Einhäupl K. Cefotaxime vs penicillin G for acute neurologic manifestations in Lyme borreliosis. A prospective randomized study. Arch Neurol. 1989; 46:1191-4.

(72) Kohlhepp W, Oschmann P, Mertens H-G. Treatment of Lyme borreliosis. Randomized comparison of doxycycline and penicillin G. J Neurol. 1989; 236:464-9.

(73) Pfister H, Preac-Mursic V, Wilske B, Schielke E, Sörgel F, Einhäupl K. Randomized comparison of ceftriaxone and cefotaxime in Lyme neuroborreliosis. J Infect Dis. 1991; 163:311-8.

(74) Karlsson M, Hammers-Berggren S, Lindquist L, Stiernstedt G, Svenungsson B. Comparison of intravenous penicillin $\mathrm{G}$ and oral doxycycline for treatment of Lyme neuroborreliosis. Neurology. 1994; 44:1203-7. 
(75) Roháčová H, Hanči J, Hulinská D, Mailer H, Havlík J. Ceftriaxone in the treatment of Lyme neuroborreliosis. Infection. 1996; 24:90-2.

(76) Borg R, Dotevall L, Hagberg L, Maraspin V, Lotric-Furlan S, Cimperman J, et al. Intravenous ceftriaxone compared with oral doxycycline for the treatment of Lyme neuroborreliosis. Scand J Infect Dis. 2005; 37:449-54.

(77) Ljøstad U, Skogvoll E, Eikeland R, Midgard R, Skarpaas T, Berg A, et al. Oral doxycycline versus intravenous ceftriaxone for European Lyme neuroborreliosis: a multicentre, non-inferiority, double-blind, randomised trial. Lancet Neurol. 2008; 7:690-95.

(78) Shadick NA, Phillips CB, Logigian EL, Steere AC, Kaplan RF, Berardi VP, et al. The long-term clinical outcomes of Lyme disease. A population-based retrospective cohort study. Ann Intern Med. 1994; 121:560-7.

(79) Shadick NA, Phillips CB, Sangha O, Logigian EL, Kaplan RF, Wright EA, et al. Musculoskeletal and neurologic outcomes in patients with previously treated Lyme disease. Ann Intern Med. 1999; 131:919-26.

(80) Klempner MS, Hu LT, Evans J, Schmid CH, Johnson GM, Trevino RP, et al. Two controlled trials of antibiotic treatment in patients with persistent symptoms and a history of Lyme disease.

N Engl J Med. 2001; 345:85-92.

(81) Kaplan RF, Trevino RP, Johnson GM, Levy L, Dornbush R, Hu LT, et al. Cognitive function in post-treatment Lyme disease: do additional antibiotics help? Neurology. 2003; 60:1916-22.

(82) Krupp LB, Hyman LG, Grimson R, Coyle PK, Melville P, Ahnn S, et al. Study and treatment of post Lyme disease (STOP-LD): a randomized double masked clinical trial. Neurology. 2003; 60:1923-30.

(83) Fallon BA, Keilp JG, Corbera KM, Petkova E, Britton CB, Dwyer E, et al. A randomized, placebo-controlled trial of repeated IV antibiotic therapy for Lyme encephalopathy. Neurology. 2008; 70:992-1003.

(84) Walsh CA, Mayer EW, Baxi LV. Lyme disease in pregnancy: case report and review of the literature. Obstet Gynecol Surv. 2007; 62:41-50.

(85) Mylonas I. Borreliosis during pregnancy: a risk for the unborn child? Vector Borne Zoonotic Dis. 2011; 11:891-8.

(86) Smith GN, Gemmill I, Moore KM. Management of tick bites and lyme disease during pregnancy. J Obstet Gynaecol Can. 2012; 34:1087-91.

(87) Nadelman RB, Hanincová K, Mukherjee P, Liveris D, Nowakowski J, McKenna D, et al. Differentiation of reinfection from relapse in recurrent Lyme disease. N Engl J Med. 2012; 367:1883-90.

(88) Nowakowski J, Nadelman RB, Sell R, McKenna D, Cavaliere LF, Holmgren D, et al. Long-term follow-up of patients with culture-confirmed Lyme disease. Am J Med. 2003; 115:91-6.

(89) Krause PJ, Foley DT, Burke GS, Christianson D, Closter L, Spielman A; Reinfection and relapse in early Lyme disease. Tick-Borne Disease Study Group. Am J Trop Med Hyg. 2006; 75:1090-4.

(90) Nadelman RB, Wormser GP. Reinfection in patients with Lyme disease. Clin Infect Dis. 2007; 45:1032-8.

(91) Jares TM, Mathiason MA, Kowalski TJ. Functional outcomes in patients with Borrelia burgdorferi reinfection. Ticks and tick-borne dis. 2014; 5:58-62.

(92) Kalish RA, McHugh G, Granquist J, Shea B, Ruthazer R, Steere AC. Persistence of immunoglobulin M or immunoglobulin $\mathrm{G}$ antibody responses to Borrelia burgdorferi 10-20 years after active Lyme disease. Clin Infect Dis. 2001; 33:780-5. 